\title{
O Storytelling como ferramenta de aprendizado ativo
}

\section{Storytelling as an active learning strategy}

DOI: $10.21530 /$ ci.v14n2.2019.917

\author{
Marcelo M. Valença ${ }^{1}$ \\ Ana Paula Balthazar Tostes²
}

\section{Resumo}

Este artigo destaca a prática do storytelling com o objetivo de analisar sua conexão com a metodologia de aprendizado ativo. Para tanto, apresentamos os debates sobre aprendizado ativo, sua finalidade, estratégias e métodos para apontar as contribuições do storytelling a essa metodologia. Os principais objetivos do artigo são: esclarecer e evidenciar as diferenças entre a atividade de contar histórias como forma de entretenimento e a utilidade dessa prática como estratégia pedagógica, no contexto do aprendizado ativo em áreas em que não tem sido usada, como nas Relações Internacionais. Analisamos a incorporação da prática do storytelling pela metodologia do aprendizado ativo em diferentes níveis de ensino. Concluímos que, dentre as suas contribuições possíveis, destacam-se a retenção de conhecimento no longo prazo e a construção de conexões cognitivas entre o objeto de estudo e a realidade social dos estudantes, podendo promover aprendizado pela empatia e capacidade de autonomia na atitude ativa em busca de solução de problemas, levando-se em conta contextos sociais de diferentes níveis: local, nacional e, também, internacional.

Palavras-chave: Aprendizado Ativo; Storytelling; Narrativas; Cognição; Aprendizagem e Ensino em Relações Internacionais.

1 Doutor em Relações Internacionais (PUC-Rio). Professor Adjunto da Escola de Guerra Naval e do Programa de Pós-Graduação em Estudos Marítimos (PPGEM). Coordenador do Laboratório de Métodos de Ensino Inovadores (LabMEI/EGN) e do Laboratório de Ensino e Pesquisa em Relações Internacionais (LabRI/UERJ).

2 Doutora em Ciência Política (IUPERJ) e pós-doutorado pela USP. Professora do Programa de Pós-Graduação em Relações Internacionais (PPGRI/UERJ), coordenadora do Curso de Relações Internacionais e do Laboratório de Ensino e Pesquisa em RI (LabRI/UERJ). Foi professora em Michigan State University (MSU) e pesquisadora visitante na Universidade Livre de Berlim (ULB).

Artigo submetido em 18/03/2019 e aprovado em 01/08/2019. 


\begin{abstract}
This article focuses on the practice of storytelling to investigate its connections with the methodology of active learning. In order to do so, we introduce the debate on active learning, its purposes, strategies, and methodologies to highlight the contributions from the use of storytelling as a pedagogical tool. The main objectives of the article are: to clarify and highlight the differences between the activity and practice of storytelling as a form of entertainment and the usefulness of this practice in areas where it has not been used, such as in International Relations. We investigate the incorporation of the practice through the methodology of active learning and in what ways make storytelling a primordial element in the constitution of the learning environment at different levels of education. We conclude that among its contributions are the retention of knowledge in the long term and the construction of significant connections between the object and social reality of students, which may generate interaction between contexts on different levels: local, national and international.
\end{abstract}

Keywords: Active Learning; Storytelling; Narratives; Cognition; Teaching and Learning in International Relations.

\title{
Introdução
}

Contar histórias sempre foi uma forma de transmitir conceitos, valores, ideias e imagens sobre o mundo e as experiências humanas. Mitos e personagens, reais ou fictícios, resgatam elementos para construir, reproduzir e disseminar narrativas que produzam significado às audiências, a partir de seus contextos social e cultural. Como prática social, o ato de contar histórias é ancestral (Heinemeyer, 2018), já que não conhecemos épocas ou sociedades em que tal atividade não estivesse presente. Consequentemente, sua inserção e valorização como ferramenta no campo dos estudos da educação superior se reflete no contexto da recuperação e revalorização do conhecimento tradicional, da reprodução de cultura, valores, preceitos morais e sentidos do mundo.

É nesse contexto que se insere o storytelling como ferramenta pedagógica. Ele consiste, grosso modo, no uso de narrativas com significado social ou cultural para promover a reflexão acerca de conceitos e valores, de forma a consolidar essas ideias abstratas por meio da percepção da relevância e significância de tais conceitos e valores a um grupo de indivíduos. ${ }^{3}$ Sua combinação com métodos

3 Como apontamos na segunda seção, “contação” de histórias e storytelling são técnicas distintas e apresentam diferenças significativas em suas estruturas e propósitos. Optamos por não traduzir storytelling justamente para 
de aprendizado ativo permite estimular processos de motivação e construir significados por meio de narrativas em campos do saber em que seu uso tem sido restrito, como nos cursos de Relações Internacionais. Isso porque, como pontuamos, o contexto social, normalmente explorado pelo uso da prática do storytelling, parece mais adequado a práticas pedagógicas no campo de estudos antropológicos, da História ou outros saberes que valorizam a cultura local e a tradição. O desafio deste artigo é esclarecer a prática do storytelling e seu uso instrumental, no contexto de uma metodologia ativa de aprendizado, sugerindo sua utilidade para o ensino na área de Relações Internacionais e, de forma mais ampla, na Ciência Política.

A lógica subjacente à utilização dos recursos de aprendizado ativo é que o estudante se torna parte ativa na construção do conhecimento (POWNER; ALLENDOERFER, 2008; KILLE, KRAIN; LANTIS, 2010; INOUE; KRAIN, 2014). Nesses termos, considera-se que o estudante assume papel tanto como receptor quanto criador de conhecimento, em uma lógica semelhante à proposta pelo “Paradigma do Aprendizado”. Este, tal como descrito por Robert Barr e John Tagg (1995), parte da distinção entre ensino e aprendizado, para concluir que aprender não equivale a reproduzir o que é informado. ${ }^{4}$ Aprender envolve analisar a informação criticamente e, com isso, interagir e dar sentido a ela, conectando o conhecimento à experiência. Objetivos semelhantes podem ser buscados por meio de outras metodologias de ensino. Mas, no caso do aprendizado ativo, há a preocupação de envolver o estudante no processo pedagógico, para que ele possa compreender tais etapas e entender o porquê dessas escolhas. Por essa razão, o aprendizado ativo vem se confundido com uma prática simples, isolada ou não organizada. No entanto, e como apontamos, a literatura sobre aprendizado ativo tem se debruçado sobre uma aprofundada sistematização de sua instrumentalização.

O storytelling é encontrado na formação social e cultural da educação espontânea ao longo de séculos e em diferentes comunidades e culturas (ENGERT; SPENCER, 2009; ANDREWS; HULL; DONAHUE, 2009; HEINEMEYER, 2018), mas a estrutura das etapas para o papel de seu cumprimento é fruto de considerações

manter a diferença entre a prática de contar histórias fora de um contexto didático e o ato de contar histórias como estratégia em um ambiente pedagógico mais amplo de aprendizado ativo.

4 De acordo com a literatura, o que chamamos de Paradigma do Aprendizado (Learning Paradigm) se contrapõe ao modelo proposto pelo Paradigma do Ensino (Instruction Paradigm). Este, que reproduz o modelo tradicional de aprendizado, pressupõe que os alunos aprendam a partir de uma fonte de autoridade que propagaria o conhecimento, unilateralmente, por meio de palestras expositivas ou leituras. O aluno comprovaria que aprendeu a partir da internalização e repetição, sem questionar o conteúdo aprendido. 
e estudos mais recentes. A tradição oral se serve da atividade de contar histórias, mas o uso do storytelling em ambientes ativos de aprendizagem se inspira na tradição oral e soma a ela práticas e ferramentas para a representação e perpetuação de estruturas mentais e morais de comunidades, em ambientes pedagógicos planejados. Seu uso reflete a experiência empática com espaços e contextos de valores tradicionais alternativos e sociedades diversificadas. A metodologia de aprendizado ativo opera como estrutura para a prática da "contação" de histórias ganhar a dimensão do storytelling.

O storytelling é uma prática que se utiliza de recursos de memória, importância do folclore, da transmissão de valores e uso de personagens - que podem ser líderes políticos, culturais ou religiosos. Narrativas como mitos, contos e lendas, descrevem lições de moral e comportamento, reforçam costumes e reproduzem símbolos por meio de uma estrutura argumentativa, permeada por instrumentos de persuasão e comunicação. No entanto, diferentemente da tradição oral de contar histórias, o storytelling pressupõe que a audiência reaja à narrativa, participando ativa e conjuntamente da construção da aprendizagem.

Nesse contexto, o storytelling passa a funcionar como uma estratégia direcionada ao aprendizado. Estudos sobre a inclusão da narrativa no processo educacional levaram ao desenvolvimento de uma estrutura de etapas e modelo que consiste em incorporar elementos pedagógicos que motivam a criatividade, a interação e o senso crítico daqueles que seriam apenas a audiência de uma história contada de modo tradicional. No campo das Relações Internacionais, o uso da narrativa e de estratégias de aprendizado ativo serve para gerar senso crítico e provocar reflexões na busca de interferência no mundo. Além disso, proporciona a construção de empatia com outros contextos e valores que não se encontram, de fato, tão separados ao considerarmos as relações entre sociedades, grupos e interseções de identidades e temas transnacionais e transversais na história das sociedades. Nesse caso, o campo social transnacional pode ser visto como próprio ao estudante e não como outro lugar distante, sem inserção, escuta ou olhar adequado. O storytelling torna-se uma ferramenta de empoderamento dos estudantes de Relações Internacionais para saírem do papel de expectadores dos eventos no mundo e se perceberem como polos ativos e participantes do processo de continuidade e/ou mudança.

Estruturamos nosso argumento em três seções, além desta introdução e da conclusão. Na primeira, apresentamos a revisão da literatura sobre aprendizado ativo e a definição dos parâmetros desta metodologia. Na segunda seção, 
identificamos algumas das razões para a falta de compreensão sobre a metodologia de aprendizado ativo 5 e o storytelling, contextualizando-se suas etapas, características, instrumentos e finalidades alternativas aos modelos tradicionais. Na terceira seção exploramos algumas das vantagens proporcionadas pelo storytelling como ferramenta de aprendizado e de ensino. Indicamos sua adequação e contribuições para a constituição de um ambiente pedagógico em sala de aula, fomentando empatia entre os alunos e entre alunos e professor. Assim, apontamos, também, as contribuições que a ferramenta traz para a relação entre professor e aluno no processo de aprendizagem e de ensino, como a retenção de conhecimento no longo prazo e a construção de conexões significativas entre o objeto de estudo e a realidade social dos estudantes. Na conclusão, apresentamos considerações complementares sobre o uso do storytelling e sua pertinência para a metodologia de aprendizado ativo. Sugerimos que o recurso a ferramentas tradicionais pode ser combinado à metodologia de aprendizado ativo a partir de sua inserção nos objetivos pedagógicos a serem alcançados, além da própria busca por um conhecimento crítico e relevante socialmente, tanto para estudantes quanto para profissionais de ensino.

\section{Contribuições do aprendizado ativo ao processo pedagógico}

O aumento recente de espaços para discussões sobre metodologias de aprendizado no campo das Relações Internacionais no Brasil proporcionou um ambiente saudável e necessário ao debate sobre o tema. No tocante à produção de conhecimento, o maior envolvimento de acadêmicos da área de Relações Internacionais nesses debates impulsionou publicações no país e no exterior. O lançamento de dossiês temáticos em periódicos e de livros editados aponta o esforço e a percepção sobre a necessidade de se partilhar o conhecimento produzido, sob diferentes formas, mecanismos e perspectivas (PINHEIRO; VEDOVELI, 2012; CARVALHO, 2013; INOUE; KRAIN, 2014; CARVALHO, 2014; VALENC A A INOUE 2017; DRI et al., 2017; ZANELLA; NEVES, 2017; CHAGAS-BASTOS; BURGES, 2018 ).

5 O exemplo mais recorrente é o do uso de filmes em sala de aula. A mera exibição de um filme serve para ilustrar um aspecto ou um ponto específico do tema em estudo, o que não invalida de forma alguma o seu uso. Contudo, isso não implica a extensão de sua capacidade pedagógica quando é conduzido fora de um contexto mais amplo de método de aprendizagem ativa. Conforme expomos, a opção pelo método de aprendizagem ativa pressupõe sua incorporação deliberada no planejamento do curso e da aula, com suas motivações e objetivos apresentados, a priori, aos alunos. 
Encontramos na produção de acadêmicos brasileiros, ainda, trabalhos com abordagens tangentes à didática, como estudos sobre o desenvolvimento do campo das Relações Internacionais no Brasil, tanto na graduação quanto na pós-graduação (SANTOS; FONSECA, 2009; FERREIRA, 2016; TOSTES; SILVA, 2017; LUCENA, 2017; FARIAS DE SOUZA; DOS SANTOS; VIEIRA, 2017).

Incentivados pela diversidade de recursos tecnológicos disponíveis e a atratividade em desenvolver atividades não-tradicionais em sala, professores em cursos de Relações Internacionais têm adotado ferramentas de aprendizado ativo para tornar seus cursos mais dinâmicos (VALENC A A INOUE, 2017; CARVALHO PINTO; CORREA; DE MEDEIROS, 2017; BADIN; CASTRO; GIANNATTASIO, 2017; INOUE; VALENC , A, 2018). Mas, em que pese esses esforços e reflexões, no que constitui o aprendizado ativo e como evidenciar seu efetivo emprego no processo de aprendizagem?

Não há definição única ou consensual para aprendizado ativo. Contudo, sua descrição, a partir das estratégias e objetivos assumidos, nos permite esclarecer seu sentido e pressupostos. O conceito de aprendizado ativo é comumente definido como um método pedagógico através do qual "estudantes se engajam em atividades como leitura, redação, discussões ou resolução de problemas que promovam a análise, síntese e avaliação do conteúdo estudado" (CENTER FOR RESEARCH ON LEARNING AND TEACHING 2016), em espaços aonde o debate e a troca de ideias sejam estimulados. Em outras palavras, há pressupostos e métodos diferenciados, desde a disposição da sala de aula, a atitude do professor em relação aos estudantes, o planejamento dos objetivos de aprendizagem, entre outros.

Apesar de popular e bastante referenciada, a metodologia de aprendizado ativo ainda é pouco explorada no Brasil. Sem desconsiderar o lugar e a importância de formas tradicionais de ensino (BURGAN; 2006; YOUNG; ROBINSON; ALBERTS, 2009; GIFKINS, 2015), destacamos que o aprendizado ativo oferece incentivos que tornam seu uso mais adequado à promoção e desenvolvimento de habilidades críticas e criativas por parte dos alunos. Percebe-se, empiricamente, um maior engajamento por parte dos estudantes em processos autônomos de busca de informação e articulação de identidades e interesses pela via de comunidades virtuais e conexões muitas vezes transnacionais. Os profissionais apegados aos métodos de ensino tradicional encontram dificuldades de interação com seus alunos, que podem ser superadas com a introdução de estratégias de aprendizado ativo. Para tanto, uma revisão da literatura internacional sobre o tema nos permite 
identificar três contribuições centrais a respeito dessa metodologia, que se traduzem em benefícios para a consolidação de um ambiente pedagógico apropriado à construção do conhecimento (POWNER; ALLENDOERFER, 2008; VALIENTE, 2008; KILLE; KRAIN; LANTIS, 2010; SHAW; GIBSON, 2010).

A primeira contribuição se refere aos ganhos cognitivos durante o processo de construção do conhecimento. O uso da metodologia de aprendizado ativo permite aos estudantes desenvolver maior capacidade de compreender o que é estudado (FOX; RONKOWSKI, 1997; JENSEN, 1998; KUZMA; HANEY, 2001). Com isso, mais do que apenas descrever o conteúdo aprendido, os alunos são capazes de criar conexões lógicas entre o tema e outras situações análogas, entre experiências vividas e à sua própria realidade.

A segunda contribuição se mostra como uma consequência da primeira. Ela se dá na relação entre a teoria e a prática, considerando a transposição do conhecimento abstrato para sua operacionalização, com enfoque em aspectos específicos a serem levados em conta no caso do ato de contar histórias como uma ferramenta de aprendizado ativo. O aprendizado ativo estimula o aluno a desafiar ideias, valores e práticas sociais pré-estabelecidas, produzindo inovação (NEWMANN; TWIGG, 2000; KILLE;L KRAIN; LANTIS, 2010), mesmo, e principalmente, quando se trata de temas controversos (LAMY, 2000). A ausência de um referencial de autoridade que emane o conhecimento permite aos alunos questionarem e explorarem formas diferentes de experimentar o conhecimento. Ou seja, sua participação constitutiva e criativa deixaria de ser uma permissividade do professor mais aberto ou tolerante à mudança dos rumos de seu planejamento acadêmico e se tornaria parte do planejado. Abre-se o espaço para contestação das bases preexistentes do saber, promovendo-se novas perspectivas sobre o tema proposto e sobre a percepção de mundo em geral. Nesse sentido, procuramos destacar, ainda, que é possível extrapolar uma visão local e presente de mundo, deixando-se de usar o recurso do storytelling como instrumento de recuperação, reforço ou "re-valoração" de cultura e identidade local e histórica, para servir como uma via de abertura e empatia com culturas alternativas, gerando uma percepção mais global e mesmo atemporal. Nesse contexto, correlações tradicionais, estruturadas a partir de um referencial epistêmico de autoridade, podem ser questionadas quanto à forma como foram construídas e como, de fato, operam junto ao tecido social.

A terceira contribuição se refere à retenção do conhecimento no longo termo. Estratégias de aprendizado ativo não apenas estimulam a memória de longo prazo (SCHACHTER, 1996), como também propiciam a interação com outras fontes de 
cognição. Conexões muitas vezes não previstas pelo educador, que só poderiam ser construídas em um ambiente horizontal de aprendizagem, encontram condições de ocorrência. Estímulos de diferentes aspectos - ler, falar, manipular - promovem maior dinamicidade ao aprendizado, aumentando a exposição e estimulando diferentes áreas do cérebro, contribuindo para a consolidação do aprendizado à longo prazo (STICE, 1986) porque é fruto de uma vivência e construção própria de sentido. Para tanto, é explorado o próprio ambiente físico de aprendizagem, como a organização da sala de aula e a disposição física e alocação de alunos e professores no espaço. O ambiente de aprendizado é repensado como mais um instrumento de auxílio na experiência de aprendizagem e na percepção intelectual, impactando o processamento daquilo que é debatido e do objetivo do conhecimento a ser alcançado (JENSEN, 1998). Não existe um lugar diferenciado para o professor, o número de estudantes é reduzido e o próprio tempo e dinâmica de aula não condizem com os padrões institucionais regulares.

Os benefícios descritos sobre a adoção do aprendizado ativo decorrem quando da utilização da metodologia em sua integralidade. Ou seja, a incorporação de estratégias isoladas só atendem ao conjunto de objetivos da metodologia quando se adequam às dinâmicas mais gerais dos objetivos pedagógicos que se busca alcançar. Fator diferencial e de primordial importância na dinâmica do aprendizado ativo é que os objetivos pedagógicos devem estar definidos previamente pelo professor e são comunicados aos estudantes de forma a propiciar a devida compreensão do papel e significado dos recursos pedagógicos utilizados. Ou seja, desde o planejamento do processo de ensino já há um compartilhamento de conhecimento sobre métodos, recursos e objetivos - o que é radicalmente diferente dos métodos tradicionais.

\section{0 aprendizado ativo como metodologia de ensino e o Storytelling como estratégia}

A premissa subjacente ao aprendizado ativo sugere que aprendemos mais quando o que é estudado faz sentido ao estar associado a uma experiência real ou mental, contextualizada por valores e sentidos culturais e sociais. $\mathrm{O}$ estudante deve perceber a relevância do tema de estudo para a sua vida ou contexto social. Assim, a ideia de se constituir um sentido mental deve ser considerada pelo professor ao planejar a atividade do storytelling. O aprendizado ativo estimula 
a criação de relações significativas originais entre estudante e objeto estudado. De certa forma, isso se reflete em maior autonomia para o estudante no que diz respeito à construção do conhecimento, visto que suas escolhas refletem e produzem impactos práticos na sua vida.

Conforme já apontado, estratégias de aprendizado ativo devem ser inseridas em um planejamento pedagógico mais amplo, proporcionando transparência aos alunos e compatibilidade aos objetivos buscados, esclarecidos desde a apresentação do curso pelo professor. Elas devem ser desenvolvidas após a definição das motivações e objetivos pedagógicos do que se planeja alcançar (BARR; TAGG, 1995; POWNER; ALLENDOERFER, 2008; KILLE; KRAIN; LANTIS, 2010; BLANTON, 2012), para que fiquem claros os propósitos e impactos esperados entre professores e alunos.

Fazendo-se uma revisão da literatura sobre o tema, classificamos quatro etapas de planejamento para o uso do método de aprendizado ativo. Elas envolvem (i) a definição dos objetivos educacionais, gerais e específicos; (ii) o planejamento e execução das atividades a serem desenvolvidas ao longo do processo de aprendizagem; (iii) o debriefing, ou seja, a análise, processamento e avaliação da experiência pelo aluno, que deve ser específico para cada atividade; e (iv) a avaliação final, fundamental para a consolidação do processo de aprendizagem esperado (KILLE; KRAIN; LANTIS, 2010; SHAW; GIBSON, 2010; VALENÇA; INOUE, 2017). Enquanto a execução de atividades acaba por constituir a parte mais visível do aprendizado ativo, as demais etapas são frequentemente negligenciadas. Contudo, o cumprimento adequado de cada etapa é de importância central para o bom resultado do processo de aprendizado ativo.

A definição dos objetivos educacionais é o que leva à definição e elaboração das atividades a serem desenvolvidas. Esta definição e sua explicitação ao aluno são aspectos que guiam estudantes e instrutores para a sua busca e construção conjunta do conhecimento. Saber o que se busca é fundamental para saber onde se quer chegar e reconhecer o momento em que se chega. Em outras palavras, alcançar os objetivos propostos e, mais importante, apontar a relevância e o propósito do que é buscado faz parte do sucesso do método. O alcance dos objetivos também é algo a ser reconhecido conjuntamente e não de forma unilateral por parte do instrutor.

A segunda etapa consiste nas atividades desenvolvidas em sala de aula. A revisão da literatura aponta que cinco tipos de atividades tendem a predominar (LAMY, 2000; NEWMANN; TWIGG, 2000; LEAN et al., 2006; DALE; PYMM, 2009; GRAYSON; DAVIES; PHILPOTT, 2009; KILLE; KRAIN; LANTIS, 2010; CARVALHO, 
2013; KRAIN; KILLE; LANTIS, 2014; BEAUPOIL-HOURDEL et al, 2017). São elas (i) o uso de estudos de caso, (ii) textos alternativos, como filmes, romances e quadrinhos, (iii) simulações e jogos, (iv) recursos tecnológicos e (v) service learning. Este consiste no aprendizado por meio do envolvimento dos estudantes em serviços ou atividades comunitárias, como angariamento de fundos, campanhas de conscientização e voluntariado. Por vezes, vemos o uso dessas atividades apartadas da estratégia geral do aprendizado ativo, de modo que seu uso não seria capaz de reproduzir os mesmos efeitos e alcançar os mesmos objetivos propostos pelo método pedagógico mais amplo (ZANELLA; NEVES, 2015) ${ }^{6}$

É durante o debriefing, referido na descrição da terceira etapa da aplicação da metodologia, que o aluno toma consciência e consolida a condição do aprendizado. O debriefing promove a reflexão sobre o que foi feito, dando significado e contexto ao processo de aprendizagem (KILLE; KRAIN; LANTIS, 2008; BLUM, 2010). A reflexão sobre o que e como se aprendeu se torna, com isso, parte integrante de todo o processo. Afinal, rever o que foi estudado e conscientemente buscar entender o papel daquele conteúdo no plano geral de aprendizado, bem como sua relevância à realidade social, consolidam o significado do conhecimento. A retenção de longo prazo do conhecimento se torna mais provável dadas as relações estabelecidas entre estudante e objeto de estudo. Finalmente, na avaliação final são revistas, coletivamente, as estratégias, que devem ser adequadas à novas perspectivas (SHAW; GIBSON, 2010).

A literatura aponta uma estrutura tripartite para o uso do storytelling, composta por um eixo central, personagens e uma perspectiva narrativa que reproduz o conhecimento proposto, a partir de determinado contexto social (ENGERT; SPENCER, 2009; SHAPIRO, 2009; JACKSON, 2016).

Como bem coloca Jerome Bruner (1986), histórias e narrativas são capazes de localizar experiências no tempo e no espaço e, com isso, atrair a atenção da audiência e conduzir ações que as conectem a partir de um contexto social. Esta capacidade aproxima o uso de histórias à premissa de que o conhecimento deve fazer sentido, não apenas intelectualmente, mas com base em sensações, recursos de memória, crítica e reflexão associados a uma experimentação. O conhecimento passa a ser ativamente constituído por estudantes e professores, na relação interativa que se estabelece, criando-se relações significativas entre estudante e objeto.

6 Sobre um panorama descritivo dessas atividades, bem como referências bibliográficas específicas para cada uma delas, ver Valença e Inoue (2017, p. 6-8). 
Apesar de se assemelhar com a exposição dissertativa de um conteúdo em sala de aula, e de sua estrutura característica remeter ao "Paradigma do Ensino" (Barr e Tagg 1995), o storytelling se adequa às premissas da metodologia de aprendizado ativo. A literatura sobre a metodologia de aprendizado ativo já reconheceu a funcionalidade e eficácia do uso do storytelling (WILLIAMS; COONEY; NELSON, 1999; MOITRA, 2014; KRAIN; KILLE; LANTIS, 2014; BEAUPOIL-HOURDEL et al., 2017).

A adoção do storytelling como ferramenta de aprendizado permite a construção de conexões de forma que as histórias contadas possam assumir diferentes papéis e funções (DAIGLE, 2016) no decorrer de um curso programado a partir da metodologia de aprendizado ativo. O professor deve estruturar sua narrativa previamente, de forma a alcançar os objetivos educacionais que estabeleceu para cada aula ou encontro específico, conforme um prévio planejamento. O uso do storytelling é moldado a partir da escolha do tema, processos e dinâmicas, com o objetivo de promover atividades de incentivo e "provocar o incômodo" necessário para que o engajamento dos estudantes ocorra de forma orientada, mas não totalmente controlada. Para tanto, o storytelling deve utilizar uma narrativa que possa ser adaptada e reformulada conforme a reação da audiência, mantendo-a atenta e participativa (DAIGLE, 2016). Para tanto, o storytelling é uma ferramenta que deve se adequar às reações e respostas aos estímulos da audiência, sempre se levando em conta o contexto social ao qual se insere o processo pedagógico. Quando não se encontram presentes esses elementos, pode-se comparar a atividade de se contar histórias com uma pedagogia mais tradicional. Nesse sentido é que, no caso do aprendizado ativo, deve-se considerar a relevância e o papel do cumprimento das etapas expostas anteriormente.

Espera-se que o empenho do professor leve ao engajamento da audiência em função da provocação de emoções, questionamentos, novas ideias e imaginações derivadas da experiência do storytelling. Podemos dizer que as reações também moldam a história, que os sentidos percebidos e sua experiência não se descolam da narrativa; tudo se torna uma só atividade integrada. A partir da combinação entre narrador e audiência, o professor deve ser capaz de realizar a intermediação entre a história em si e a audiência, em que se espera dele a flexibilidade na condução da atividade de forma aberta, criativa e provocadora.

No contexto do aprendizado ativo, o uso do storytelling surge ainda como uma estratégia versátil e complementar a outras estratégias de aprendizado. Seu papel como estratégia de aprendizado ativo encontra apoio, por exemplo, na sua 
aplicação junto a elementos de cultura popular, como a partir de sua inserção em role playing games e em videogames (HAYDEN, 2010; KUCHINSKY, 2010; BLANTON, 2012; CARVALHO, 2014). Esse é um novo uso, mas que nos permite conectar elementos e sentidos associados à construção de conhecimento, através de meios que espelham a própria cultura atual.

\section{Estruturas narrativas e contribuições ao aprendizado}

De modo geral, no contexto da pedagogia formal e mesmo na educação informal, a "contação" de histórias constitui uma das atividades mais tradicionais de ensino ao longo do tempo e em diferentes culturas (WILLIAMS; COONEY; NELSON, 1999; HEINEMEYER, 2018). Esse método é utilizado em diferentes disciplinas e níveis de instrução com razoável sucesso (HEINEMEYER; DURHAM, 2017, p. 36-41), graças aos benefícios proporcionados ao processo de aprendizado. O uso de narrativas como ferramenta de ensino, no contexto da educação formal, tem sido considerado uma forma eficiente de tornar acessível experiências de aprendizagem aos estudantes, sempre que exijam abordagens mais relacionadas à construção de sentidos e significados do que à transmissão de conceitos ou informações. Temas, conteúdos e objetivos pedagógicos, que poderiam sofrer eventuais ruídos dado o grau de complexidade, interdisciplinaridade ou nível de abstração, ganham novas dimensões de agregação do interesse, construção de identidades e empatia.

Como apontamos, o aprendizado se torna mais efetivo quando inserido em um contexto social que dá significado e aplicabilidade ao que se aprende. Ao prover o contexto, limites e inserção da informação em situações práticas, a narrativa no storytelling permite que a audiência compreenda melhor o grau de relevância, abrangência e mesmo utilidade do conhecimento em foco (SCHANK, 1982; SCHANK; ABELSON, 1995).

É recorrente, no uso da ferramenta do storytelling, a construção de histórias em uma estrutura narrativa tripartite, em um modelo designado como a "jornada do herói” (CAMPBELL, 2008). Na jornada, o herói é o protagonista da narrativa. Sua visão de mundo é constituída a partir de seus ideais e valores, promovendo um conhecimento parcial do ambiente que o cerca. A audiência consegue se identificar com o herói porque ele oferece os traços de humanidade comuns aos membros daquele grupo social. Nesta primeira parte da história é apresentado 
o universo do herói, antes do início de sua aventura a ser contada. Trata-se do momento em que a audiência conhece o herói, seu contexto e suas crenças iniciais.

A segunda parte envolve a audiência no desafio lançado ao herói, promovendo impactos significativos no mundo como é conhecido. O mote subjacente a essa etapa se caracteriza por um dilema ou confrontação posto diante do herói. O herói é desafiado e o mundo que ele conhece é questionado, trazendo dúvidas para a sua própria continuidade.

Finalmente, a terceira parte envolve a redenção do herói, quando a vitória ou conquista é alcançada, com impactos sobre o mundo que o protagonista conhece. Ele supera o dilema que lhe é imposto, mas o mundo que conhece é alterado pela sua experiência recente. A lição moral da história implica, necessariamente, na transformação do próprio herói, ou do seu mundo, de forma a refletir essa mudança.

Essa estrutura narrativa é comum e considerada clássica na proposta do uso do storytelling, sempre enquanto uma ferramenta no contexto de uma metodologia de aprendizado ativo. Porém, não é a única possível.

Naturalmente as histórias escolhidas podem ter ambientações atraentes à construção de sentidos, seja em ambientes que valorizam elementos fantásticos ou da realidade urbana, da comunidade, de uma estrutura de valores e preocupações vistos como legítimos, tais como ameaças ao meio ambiente ou violência, por exemplo. As narrativas, construídas pelo contador de histórias, são adaptáveis ao público para o qual são apresentadas, podendo ser moldadas conforme o seu interesse, grupo social ou idade e, até mesmo, a partir da maior ou menor aceitação da audiência ao que é falado.

Nessa lógica, alguns elementos podem ser enfatizados em detrimento a outros, conforme a audiência. Roteiros estruturados de forma semelhante podem servir a histórias diferentes. Podem-se construir narrativas diversas para compor elementos e conteúdos de diferentes maneiras, mantendo-se sua estrutura e premissas, mas destacando-se elementos distintos, conforme os objetivos pedagógicos e sociais a serem atingidos.

A escolha do storytelling implica uma expectativa de que haja compreensão da história e também a geração de uma estruturação mental da narrativa (ROBERTS, 2006, p. 704), de forma que sua assimilação faça parte de um processo ativo de compreensão, crítica, reflexão e aprendizado. A ferramenta estimula e demanda sensações visuais da audiência, de modo que sua participação extrapole a mera passividade de um espectador. Configurando-se, portanto, em uma maneira de 
compartilhar e transmitir experiências, sem que haja qualquer forma de autoridade epistêmica entre o contador e sua audiência. Esse é um ponto primordial para se compreender a função e o potencial do storytelling: o fato de que se quebra a relação de autoridade na relação de aprendizagem e se valoriza a contribuição na narrativa, na busca da solução dos desafios e no desfecho das histórias. Histórias narradas que se espelham no mundo vivido.

A revisão da literatura sobre storytelling aponta diversos benefícios aos professores advindos de seu uso em sala de aula (WILLIAMS; COONEY; NELSON, 1999; ANDREWS; HULL; DONAHUE, 2009; MOITRA, 2014; BEAUPOIL-HOURDEL et al., 2017; DAMAYANTI, 2017). Entre eles estão (i) o empoderamento do narrador e da audiência (desfazendo-se a relação verticalizada entre autoridade que ensina e espectador que recebe a informação), (ii) a possibilidade de destacar aspectos específicos para cada audiência e objetivo, (iii) os elementos dinâmicos à condução da aula e (iv) a contribuição que o storytelling oferece para sustentar conceitos abstratos e/ou complexos, aproximando-os da audiência e de sua realidade e contexto social.

Ainda que estes elementos dialoguem com os benefícios proporcionados à audiência, os impactos do uso do storytelling podem ser analisados à luz das contribuições ao professor, em função das possibilidades de alcançar os objetivos pedagógicos buscados. Outro aspecto a ser destacado é que, considerando-se que a audiência deve se sentir estimulada a se engajar no processo de storytelling, a habilidade do professor em adaptar sua narrativa, para se adequar a interesses pedagógicos e que atendam ao grupo, aparece como elemento necessário para o sucesso de uma participação ativa e para a consequente construção do conhecimento que se espera. Mais ainda, o professor deve conduzir o processo sem se prender a modelos pré-estabelecidos. Importante que o instrutor se sinta livre para inovar na sua prática docente. Percebendo atalhos e espaços para engajar seus alunos por meio do estímulo de seus interesses, o professor deve estar confortável para criar ambientes pedagógicos propícios ao aprendizado, ser aberto a contribuições inesperadas e, por vezes, tem que lidar com problemas não antecipados.

Além disso, a perspectiva de algo novo a ser ofertado aos estudantes e a clareza nos objetivos propostos fazem com que o professor ganhe maior liberdade de ação, possa desenvolver novas habilidades pedagógicas e usar sua criatividade de formas mais diversas e particulares. Esses benefícios se estruturam lógica e progressivamente, não excluindo, a priori, outras contribuições que o storytelling, como ferramenta de ensino, proporcionam. Contribuições não mapeadas na 
literatura podem ser ainda percebidas e destacadas. Exemplos são incentivos à melhoria da performance do professor em sala, dando a ele maior desenvoltura e empatia junto aos estudantes e maior diálogo com recursos apurados a partir de temas culturais e valores. Pode-se considerar, ainda, o emprego mais efetivo de meios relativamente tradicionais de estímulo à aprendizagem, tais como o uso de filmes, romances, quadrinhos, jogos, etc. Lembramos que, no caso do aprendizado ativo, não basta o uso dos recursos, mas a sua associação às técnicas propostas pela metodologia mais ampla, conforme já abordado neste artigo. Finalmente, a interdisciplinaridade, como elemento para evidenciar a importância do tema de conhecimento abordado no processo ensino-aprendizagem para a vida e carreira dos estudantes, também é reforçada, assim como no ensino tradicional contemporâneo.

É natural imaginar que escolhas de métodos ou estratégias a serem utilizadas em sala de aula passam por um cálculo de custo e benefício dos professores. Assim, o grau de liberdade do professor deve estar associado à sua postura responsável sobre a decisão de suas escolhas e pelo planejamento de atividades. Com isso, e em consonância com a ideia de explicar por que determinadas ferramentas são utilizadas em detrimento a outras, é importante se debruçar, também, sobre os impactos da aplicação do storytelling como ferramenta pedagógica junto aos estudantes. Partindo do pressuposto que a metodologia de aprendizado ativo incentiva e se sustenta na colaboração entre professor e aluno para a descoberta e construção do conhecimento, os alunos também se veem beneficiados no seu aprendizado com o uso dessa ferramenta. Aprendem não apenas o conteúdo proposto, mas a própria ferramenta de solução de problemas que serve para toda a sua vida, mesmo fora da sala de aula.

Além disso, em um contexto de aprendizado ativo, a capacidade de retenção da aprendizagem em longo termo aumenta, pois a memória constituída é dotada de diversos recursos de imagem, problematização, opinião, conceitos e participação na narrativa. Não à toa, somos capazes de lembrar de eventos e fatos quando inseridos em uma narrativa que afete a capacidade de conexões, sensações, memórias e contexto social, semelhante ao que ocorre com histórias contadas.

As contribuições do storytelling aos alunos envolvem a exposição dos conceitos e ideias à audiência por mais de um formato, sem se limitarem apenas a isso, pois o storytelling proporciona o aprendizado a partir do envolvimento direto com o problema e uma interação com seus desafios e questões. Ao entenderem como a trama se desenvolve e como os elementos se conectam socialmente, seja 
pela associação às suas próprias vivências ou à busca por soluções, o estudante aprende enquanto reflete sobre sua atividade. Nesse sentido, tal como ocorre com o instrutor, o storytelling proporciona o empoderamento da audiência. Ao reconhecer os valores narrados à sua experiência, os alunos se engajam com o conteúdo e, portanto, aumentam as possibilidades de fixar os conhecimentos trabalhados.

Ao lembrar e conectar conceitos e ideias a aplicações no seu contexto social, o processo de aprendizado se torna mais efetivo porque os alunos entendem a lógica subjacente ao conteúdo. Valendo destacar, como este artigo procura também contribuir, que o contexto social em foco pode ter diferentes camadas e níveis de identificação. Ao considerarmos o uso desta ferramenta para a geração de estudantes conectados internacional e transnacionalmente, ideias e valores sociais não perpassam mais, necessariamente, uma experiência geograficamente limitada. Ou, em outros casos, a distância geográfica não é capaz de desconstituir a importância da empatia no aprendizado sobre valores e visão crítica do mundo. Assim, a ferramenta do storytelling pode ser de grande utilidade em um curso de Relações Internacionais: temas como meio ambiente, direitos humanos e direitos de refugiados, dentre outros, podem ser largamente explorados, considerando-se um contexto social não limitado aos costumes locais, regionais ou nacionais.

Finalmente, o storytelling leva à criação de conexões entre o estudante e o conteúdo analisado, dando sentido ao que é estudado e levando a dois impactos principais. O primeiro se dá em razão da proximidade dos conceitos com as experiências pessoais, estimulando a capacidade de resolução de problemas e de inovação, criando possibilidades para serem aplicadas em cenários semelhantes no futuro. O estímulo à inovação não se dá, assim, apenas na resolução de problemas por parte do aluno: a literatura aponta que há uma busca por melhores práticas, em conjunto com o narrador, para resolver questões específicas da narrativa.

O segundo impacto que se destaca é a maior autonomia do estudante no que tange às suas escolhas acerca do "que" e "como" aprender. O storytelling permite, pois, ênfase em aspectos desejados e promoção de competências autônomas no aprendizado. O conhecimento passa a ser direcionado a questões particulares e relevantes, proporcionando maior interesse e praticidade ao aplicar técnicas na resolução de problemas existentes ou futuros.

Sendo o storytelling uma forma de ensinar e aprender sobre cultura e identidade, uma extrapolação sobre essa função pode sugerir impactos no que tange ao aprendizado de práticas e valores do contexto social dos participantes do 
processo. Estudantes jovens e conectados com outros grupos sociais e com redes transnacionais de comunicação e de identificação também podem imaginar novas perspectivas e leituras da realidade, a partir de um contexto concreto pré-existente e transformar experiências que conhecem de acordo com o que escutam. No caso específico do storytelling, essas modificações levam em conta tanto a empatia quanto as experiências compartilhadas - assim como o formato da jornada do herói pode facilitar a estruturação do aprendizado e sua compreensão em termos significativos.

\section{Considerações sobre o tema e possibilidades para o futuro}

Ao longo do artigo, destacamos qualidades necessárias ao professor engajado na metodologia do aprendizado ativo, na medida em que o método não tradicional pressupõe uma responsabilidade do professor em conduzi-lo, de forma a alcançar seus objetivos pedagógicos. Etapas devem ser cumpridas e expectativas do profissional de ensino podem ser superadas.

Como apontado na seção em que revisamos a literatura de aprendizado ativo, a exposição de um conteúdo por meio de diferentes estímulos implica que o professor possua qualidades e vontade direcionadas ao emprego da metodologia. Isso porque é preciso levar em conta que a cognição humana se estrutura a partir de conexões, muitas vezes particulares, que unem os diferentes tipos de informações a que somos expostos e que se organizam a partir de modelos e formatos que nos são familiares.

O storytelling, como ferramenta de aprendizagem, oferece também a oportunidade para aprendizado com base nas experiências vividas tanto pelo contador de história, quanto pela audiência ativa e pela comunidade social na qual se inserem professores e alunos. Não obstante, as próprias experiências dos alunos podem dialogar com o que é transmitido pela narrativa, constituindo e reconstituindo valores e ideias, desde uma atitude proativa no processo de aprendizado. A reflexão se dá a partir da problematização de valores e ideias, experiências e conhecimentos. Em suma, o storytelling traz uma dimensão de empoderamento às partes envolvidas em um processo de aprendizado, fazendo com que os atores colaborem na descoberta do conhecimento e de sua adequação às necessidades, convergindo para um aprendizado colaborativo, crítico e socialmente relevante. 
A valorização da metodologia e sua utilização por instituições de ensino superior tem crescido no Brasil nos últimos anos. Indicativos dessa tendência podem ser percebidos tanto a partir de iniciativas institucionais, acadêmicas, como no âmbito da produção de conhecimento informal. No campo das instituições profissionais que promovem diálogos nacionais e internacionais importantes sobre a produção de conhecimento e pesquisa no campo da Ciência Política e das Relações Internacionais no Brasil, podemos apontar a criação de áreas temáticas específicas nas duas principais associações desses profissionais ${ }^{7}$ (RAMANZINI JÚNIOR; LIMA, 2017). Tais associações possuem publicações e promovem encontros nacionais. Mais especificamente, em termos de instituições de ensino superior, podem-se apontar iniciativas concretas na adoção de estratégias de aprendizado ativo em cursos de graduação como as da Fundação Getúlio Vargas, no Rio de Janeiro, que estruturou salas de aula especiais em formato apropriado ao uso de tal metodologia.

Contudo, o uso sistematizado de estratégias de aprendizado ativo, além do uso do storytelling no ensino superior, não são prática comum em todas as áreas de conhecimento. Uma vez sistematizado, o uso do storytelling parece ser mais facilmente aplicável, mesmo no ensino superior, em práticas pedagógicas de cursos de História, Ciências Sociais ou Antropologia, por exemplo. Isso porque seu uso é comumente relacionado ao resgate, crítica ou recondução de valores, ideias e identidades, temas relacionados à cultura e à história local. No entanto, tanto a metodologia em geral de aprendizado ativo, quanto a prática do storytelling podem contribuir decisivamente, e efetivamente o fazem, para uma relação ensino-aprendizagem em campos do saber em que o desenvolvimento de potencialidades para solução de problemas é valorizado, como as engenharias (ALMEIDA et al,, 2019). Neste artigo, enfatizamos a utilidade e os benefícios do storytelling na formação em Relações Internacionais, considerando-se contextos sociais em diferentes níveis. Dessa forma, procura-se primeiramente conceituar e contextualizar o storytelling para depois associar e sugerir tal ferramenta metodológica como adequada para o ensino superior em campos do saber em que não é explorada suficientemente, como no campo das Relações Internacionais.

A inclusão de discussões sobre metodologias de ensino e experiências em sala de aula no ensino superior, no campo das Relações Internacionais, no entanto,

7 No âmbito da Associação Brasileira de Ciência Política (“ABCP”), a Área Temática de Ensino e Pesquisa foi criada em 2010, enquanto no da Associação Brasileira de Relações Internacionais (“ABRI”), a criação da Área Temática de Ensino, Pesquisa e Extensão se deu no ano de 2015. 
é recente e menos comum do que em áreas em que o foco de discussão sobre cultura, valores e identidades aparece de forma mais evidente. No caso dos estudos internacionais, o que motivou esse artigo foi identificar a importância da prática também para uma área em que o contexto social importa, mesmo que seja levado em conta o nível internacional e transnacional de produção de valores, de história, e de necessidade de se estimular a empatia e co-constituição de conhecimento e reflexão na busca de solução de problemas.

Enfim, o potencial de uso do storytelling como ferramenta de aprendizado é vasto, ainda que nem sempre ou por si só cumpra o objetivo contextualizado pela metodologia do aprendizado ativo. Ainda que haja uma tradição que associa a “contação" de histórias e o storytelling a uma vertente mais tradicional de aprendizado, trata-se de uma ferramenta poderosa, com potencial para contribuir com as estratégias de aprendizado ativo, inclusive no campo dos estudos internacionais e da política.

O artigo não buscou apresentar casos ou exemplos empíricos do uso da prática e da metodologia, mas reforçar aspectos conceituais e teóricos sobre metodologias de ensino, que abrem espaço para o debate a respeito do valor e utilidade do storytelling em cursos em que esta prática não tem sido usada com frequência no Brasil, comparativamente ao campo, por exemplo dos estudos antropológicos. Histórias de heróis reais como Chico Mendes, no contexto do fortalecimento dos movimentos de defesa do conhecimento tradicional dos povos da floresta e preservação do meio ambiente, da menina Malala ou de Gandhi, não poderiam ser inspirações para se explorar práticas de storytelling? Seria possível a adaptação da estrutura tripartite para se explorar conteúdos e reflexões sobre tais heróis reais? Consideramos que sim. Mais ainda, consideramos que o storytelling pode ser uma ferramenta fundamental para o alcance de certos conteúdos no campo das relações internacionais.

\section{Referências}

ALMEIDA, Nival Nunes; ELMÔR FILHO, Gabriel; SAUER, Laurete Zanol; VILLAS-BOAS, Valquíria. Uma Nova Sala de Aula é Possível: aprendizagem ativa na educação em Engenharia. Rio de Janeiro: LTC, 2019.

ANDREWS, Dee H; HULL, Thomas D; DONAHUE, Jennifer A. 2009. Storytelling as an Instructional Method: Definitions and Research Questions. Interdisciplinary Journal of Problem-Based Learning v. 3, n. 2, 2009, p. 6-23. 
BADIN, Michelle Ratton Sanchez; CASTRO, Douglas; GIANNATTASIO, Arthur Roberto Capella. The Didactic Case in Teaching International Law. Meridiano 47 - Journal of Global Studies v. 18, 2017, p. 1-17.

BARR, Robert B; TAGG, John. From Teaching to Learning: a New Paradigm for Undergraduate Education. Change, 1995, p. 13-25.

BEAUPOIL-HOURDEL, Pauline; KOSMALA, Loulou; JOSSE, Helene; MASUGA, Katy; MORGENSTERN, Aliyah. When Storytelling Meets Active Learning: an Academic Reading Experiment with French MA Students.” In: SARRÉ, Cédric; WHYTE, Shona (Eds.). New Developments in ESP Teaching and Learning Research, p. 109-129, 2017. Disponível em Research-publishing.net. Acesso em: 03 jan. 2019.

BLANTON, Robert G. Zombies and International Relations: a Simple Guide for Bringing the Undead Into Your Classroom. International Studies Perspectives v. 14, n. 1, 2012, p. 1-13.

BLUM, Andrew. 2010. “Computer Simulations in the Classroom.” In DENEMARK, Robert (ed.). The International Studies Encyclopedia, p. 1-11, 2010, Blackwell Publishing. BRUNER, Jerome S. Actual Minds, Possible Worlds. Cambridge: Harvard University Press, 1986.

BURGAN, Mary. In Defense of Lecturing. Change, November/December, 2006.

CAMPBELL, Joseph. The Hero with a Thousand Faces. 3A ed. Novato: New World Library, 2008.

CARVALHO PINTO, Vânia; CORREA, Humberto; DE MEDEIROS, Fernanda. O Caso Prático Como Método De Ensino Em Teoria Das Relações Internacionais: O Programa Nuclear Iraniano Segundo Os Níveis De Análise. Meridiano 47 - Journal of Global Studies v. 18, 2017, p. 1-18.

CARVALHO, Gustavo. Virtual Worlds Can Be Dangerous: Using Ready-Made Computer Simulations for Teaching International Relations.” International Studies Perspectives v. 15 , n. 4. 2013, p. 538-557.

CARVALHO, Gustavo. "Rejoinder to 'Misusing Virtual Worlds Can Be Dangerous: a Response to Carvalho'.” International Studies Perspectives v. 15, n. 4, 2014, p. 564-565. Center for Research on Learning and Teaching. 2016. “Active Learning.” Disponível em http://www.crlt.umich.edu/tstrategies/tsal. Acesso em: 10 nov. 2016.

CHAGAS-BASTOS, Fabrício H; BURGES, Sean W Burges. The 'Briefing Note' as a Pedagogical Tool for Teaching Politics and International Relations. Journal of Political Science Education, v. 15, n. 2, 2018, p. 237-246.

DAIGLE, Megan. Writing the Lives of Others: Storytelling and International Politics. Millennium: Journal of International Studies v. 45, n. 1, 2016, p. 25-42.

DALE, Crispin; PYMM, John M. Podagogy: the iPod as a Learning Technology. Active Learning in Higher Education v. 10, n. 1, 2009, p. 84-96. 
DAMAYANTI, Ika Lestari. From Storytelling to Story Writing: the Implementation of Reading to Learn (R2L) Pedagogy to Teach English as a Foreign Language in Indonesia. Indonesian Journal of Applied Linguistics v. 6, n. 2, 2017, p. 232-245.

DRI, Clarissa Franzoi; PAGLIARI, Graciela Di Conti; LEITE, Iara; ARIENTI, Patricia Fonseca. Experiências Alternativas De Ensino Em Relações Internacionais: Experiências De Simulações E Contato Com Atores Sociais Locais Desenvolvidas Com Graduandos Da Universidade Federal De Santa Catarina. Meridiano 47 - Journal of Global Studies v. 18, 2017, p. 1-17.

ENGERT, Stefan; SPENCER, Alexander. International Relations at the Movies: Teaching and Learning About International Politics Through Film. Perspectives v. 17 n. 1, 2009, p. 83-104.

FARIAS DE SOUZA, André Luiz Coelho; DOS SANTOS, Vinicius Silva; VIEIRA, Alice Gravelle. A Internacionalização Na Formação Em Relações Internacionais No Brasil.” Meridiano 47 - Journal of Global Studies v. 18, 2017, p. 1-17.

FERREIRA, Marcos Alan S V. The Rise of International Relations Programs in the Brazilian Federal Universities: Curriculum Specificities and Current Challenges. Journal of Political Science Education, v. 12, n. 3, 2016, p. 241-255.

FOX, Richard L; RONKOWSKI, Shirley A. Learning Styles of Political Science Students. PS: Political Science and Politics v. 30, 1997, p. 732-737.

GIFKINS, Jess. “Strategies for Making Large Lectures More Interactive.” E-Ir. Publicado em: 17 out. 2015. Disponível em: http://www.e-ir.info/2015/10/17/strategies-formaking-large-lectures-more-interactive/. Acesso em: 05 fev. 2019.

GRAYSON, Kyle; DAVIES, Matt; PHILPOTT, Simon. Pop Goes IR? Researching the Popular Culture - World Politics Continuum. Politics v. 29, n. 3, 2009, p. 155-163.

HAYDEN, Craig. “Entertainment Technologies.” In DENEMARK, Robert (ed.). The International Studies Encyclopedia, p. 1-16, 2010, Blackwell Publishing.

HEINEMEYER, Catherine. "The Dying Art of Storytelling in the Classroom." The Conversation. Publicado em: 11 abr 2018. Disponível em: http://theconversation. com/the-dying-art-of-storytelling-in-the-classroom-93088. Acesso em: 01 fev. 2019. HEINEMEYER, Catherine; DURHAM, Sally. Is Narrative an Endangered Species in Schools'? Secondary Pupils' Understanding of 'Storyknowing'. Research in Education v. 99, n. 1, 2017, p. 31-55.

INOUE, Cristina Yumie Aoki; VALENÇA Marcelo M (eds.). Relações Internacionais na Sala de Aula: Ensino e Aprendizado Ativo E Outras Estórias. Campina Grande: Eduepb, 2018.

INOUE, Cristina Yumie Aoki; KRAIN, Matthew One World, Two Classrooms, Thirteen Days: Film as an Active-Teaching and Learning Tool in Cross-National Perspective. Journal of Political Science Education, v. 10, n. 4, 2014, p. 424-442. 
JACKSON, Patrick Thaddeus. 'A Long Time Ago in a Galaxy Far, Far Away....': Fantasy and Morality in Star Wars and U.S. Foreign Policy. In: Conferência Anual da International Studies Association, 2016, Atlanta (GA)..

JENSEN, Eric. Teaching with the Brain in Mind. Alexandria: Association for Supervision and Curricular Development, 1998.

KILLE, Kent J; KRAIN, Matthew; LANTIS, Jeffrey S. Active Learning Across Borders: Lessons From an Interactive Workshop in Brazil. International Studies Perspectives v. 9, n. 4, 2008, p. 411-429.

KILLE, Kent J; KRAIN, Matthew; LANTIS, Jeffrey S. "The State of the Active Teaching and Learning Literature." In DENEMARK, Robert (ed.). The International Studies Encyclopedia, p. 1-18, 2010, Blackwell Publishing.

KRAIN, Matthew; KILLE, Kent J; LANTIS, Jeffrey S. Active Teaching and Learning in Cross-National Perspective.” International Studies Perspectives v. 16, n. 2, 2014, p. $142-155$.

KUCHINSKY, Michael. “Teaching with Media.” In DENEMARK, Robert (ed.). The International Studies Encyclopedia, p. 1-16, 2010, Blackwell Publishing.

KUZMA, Lynn M; HANEY, Patrick J. And...Action! Using Film to Learn About Foreign Policy. International Studies Perspectives v. 2, n. 1, 2001, p. 33-50.

LAMY, Steven. "Teaching Introductory International Relations with Cases and Analytical Exercises.” In LANTIS, Jeffrey S.; KUZMA, Lynn M.; BOEHRER, John (eds.). The New International Studies Classroom: Active Teaching, Active Learning, Boulder e Londres: Lynne Rienner, 2000, p. 21-35.

LEAN, Jonathan; MOIZER, Jonathan; TOWLER, Michael; ABBEY, Caroline. Simulations and Games: Use and Barriers in Higher Education. Active Learning in Higher Education v. 7, n. 3, 2006, p. 227-242.

LUCENA, Andréa Freire. Produção e Difusão do Conhecimento em Relações Internacionais no Brasil: Análise de Egressos dos Mestrados. Meridiano 47 - Journal of Global Studies, v. 18, 2017, p. 1-11.

MOITRA, Karobi. Storytelling as an Active Learning Tool to Engage Students in a Genetics Classroom. Journal of Microbiology \& Biology Education v. 15, n. 2, 2014, p. 332-334.

NEWMANN, William W; TWIGG, Judythn L. Active Engagement of the Intro IR Student: a Simulation Approach. PS: Political Science and Politics v. 33, December 2000, p. 835-42.

PINHEIRO, Leticia; VEDOVELI, Paula. Caminhos Cruzados: Diplomatas e Acadêmicos na Construção do Campo de Estudos de Política Externa Brasileira. Política Hoje v. 21, n. 1, 2012, p. 211-254.

POWNER, Leanne C; ALLENDOERFER, Michelle G. Evaluating Hypotheses About Active Learning.” International Studies Perspectives v. 9, n. 1, 2008, p. 75-89. 
RAMANZINI JÚNIOR, Haroldo; LIMA, Thiago. Editorial - Challenges and Pathways of Teaching, Research and Extension in International Relations in Brazil. Meridiano 47 - Journal of Global Studies v. 18, 2017, p. 1-5.

ROBERTS, Geoffrey. History, Theory and the Narrative Turn in IR. Review of International Studies v. 32, n. 4, 2006, p. 703-713.

SANTOS, Norma Breda; FONSECA, Fúlvio Eduardo. A Pós-Graduação em Relações Internacionais no Brasil.” Contexto Internacional v. 31, n. 2, 2009, p. 353-380.

SCHACHTER, Daniel L. Searching for Memory: the Brain, the Mind, and the Past. New York: Basic Books, 1996.

SCHANK, Roger C. Dynamic Memory. Cambridge: Cambridge University Press, 1982.

SCHANK, Roger C; ABELSON, Robert P Knowledge and Memory: the Real Story. Hillsdale: Lawrence Erlbaum Associates, 1995.

SHAPIRO, Michael J. Cinematic Geopolitics. London e New York: Routledge, 2009.

SHAW, Carolyn M.; GIBSON, Kay. “Assessment of Active Learning.” In DENEMARK, Robert (ed.). The International Studies Encyclopedia, p. 1-16, 2010, Blackwell Publishing. STICE, James E. Using Kolb’s Learning Cycle to Improve Student Learning. Engineering Education v. 77, n. 5, 1986, p. 291-296.

TOSTES, Ana Paula; SILVA, Lucca Viersa. "Uma Década De Consolidação Da PósGraduação Em Relações Internacionais No Brasil (2004-2014).” Meridiano 47 - Journal of Global Studies v. 18, 2017, p. 1-16.

VALENC „A, Marcelo M.; INOUE, Cristina Yumie Aoki. “Contribuições Do Aprendizado Ativo Ao Estudo Das Relações Internacionais Nas Universidades Brasileiras.” Meridiano 47 - Journal of Global Studies v. 18, 2017, p. 1-15.

VALIENTE, Carolina. Are Students Using the 'Wrong' Style of Learning?: a Multicultural Scrutiny for Helping Teachers to Appreciate Differences. Active Learning in Higher Education, v. 9, n. 1, 2008, p. 73-91.

WILLIAMS, Karen Cachevki; COONEY, Margaret; NELSON, Jane. Storytelling and Storyacting as an Active Learning Strategy. Journal of Early Childhood Teacher Education v. 20 n. 3, 1999, p. 347-352.

YOUNG, Mark S; ROBINSON, Stephanie; ALBERTS, Phil. Students Pay Attention!: Combating the Vigilance Decrement to Improve Learning During Lectures. Active Learning in Higher Education v. 10, n. 1, 2009, p. 41-55.

ZANELLA, Cristine K; NEVES JR, Edison J. O Ensino de Relações Internacionais E O Cinema: Reflexões Sobre O Uso De Filmes Como Uma Ferramenta Pedagógica. Meridiano 47 - Journal of Global Studies v. 18, 2017, p. 1-20.

ZANELLA, Cristine K; NEVES JR, Edison J. (eds). As Relações Internacionais E O Cinema: Estado E Conflitos Internacionais. Vol. 2. Belo Horizonte: Editora Fino Traço, 2015. 